\title{
Study on the Microwave Permeability of the CNT Complex in $2-18 \mathrm{GHz}$
}

\author{
Qinghuai Li \& Xiaolai Liu (Corresponding author) \\ College of Science, University of Chemical Industry of Beijing, Beijing 100029, China \\ Tel: 86-10-6214-5859 E-mail: Liuxl1@mail.buct.edu.cn
}

The research is supported by the 863 Research Project (No.2008AB08Z513) and the School Scientific Research Project of the University of Chemical Industry of Beijing (No.B60849). (Sponsoring information)

\begin{abstract}
The frequency spectrum of the permeability of the single-walled carbon nanotubes (SWCNT)-paraffin complex is studied in $2-18 \mathrm{GHz}$, which is very important for the absorption performance of materials. The microwave absorption materials are required by strong absorption performance, wide frequency band, thin thickness, and good environmental stability. Traditional microwave absorption materials could not well fulfill above requirements. By the measurement and acquisition of the permeability paramet of SWCNT-paraffin complex in $2-18 \mathrm{GHz}$ and the Origin software under different conditions, the influence of the concentration and frequency of the complex on the permeability of the carbon nanotubes is analyzed in this article.
\end{abstract}

Keywords: Single-walled carbon nanotubes (SWCNT), Permeability, Paraffin, Microwave absorption

\section{Introduction}

Since that the Japanese electronic speculum expert S. Iijima found the CNTs in 1991 (Iijima S, 1991, P.56-86), its many advantages promoted people to study it. CNT could be regarded as the tube that the graphite flakes circle the central axes according to certain helicity, and two ends of the tube are generally sealed by the half-spherical surface gridding with pentagons (Taurisano M D, 2000, P.2022 \& Zhong, 1999, P.26-30 \& Iijima S, 1991, P.56-86 \& Bi, 2005, P.34). Each carbon atom is linked with three neighboring carbon atoms in the CNT, which will form the hexagon gridding structure, so the carbon atoms in the CNT give priority to sp2 hybridization, but the hexagon gridding structure in CNT will curve, and form the space topological structure and certain sp3 hybridization bond, so it gives priority to sp2 hybridization, with certain sp3 hybridization. The curvature of CNT with small diameter is bigger, and the proportion of sp3 hybridization is big, and contrariwise, the proportion of $\mathrm{sp} 2$ hybridization is small, and the shape change of CNT will also change the proportion of sp2 and sp3 hybridization (Ebbesen T W, 1996, P.54). CNTs generally contain single walled structure or multiple-walled structure, and they are correspondingly called as the SWCNT and MWCNT. The diameter of SWCNT is from zero point nanometer to a few nanometers, and the diameter of MWCNT is from a few nanometers to tens nanometers, and the length could achieve a few millimeters, and the fixed interval is kept among was, which equals to the walled interval of graphite, and the length could achieve tens microns, is about $0.134 \mathrm{~nm}$. The structure of MWCNT is very complex and difficult to be confirmed, and the structure of SWCNT is relatively simple, which has been deeply studied theoretically. The crystal structure of CNT is close-packed hexagonal (hcp), $a=0124568 \mathrm{~nm}, c=016852 \mathrm{~nm}, c / a=2.786$, and comparing with graphite, the value of $a$ is smaller and the value of $c$ is bigger, which indicate that CNT has stronger linkage force and higher same-axial property among atoms in the same wall of CNT, and it is the one-dimensional crystal with periodic character in the tube axial direction, and it could be regarded as the ideal one-dimensional material. For CNT, different curving modes mean different structures and different characters. When curving, the corner, i.e. the helical angle (seen in Figure 1) may occur between the hexagon gridding and the axis of CNT, and a1 and a 2 are unit vectors, $\mathrm{Ch}=n \mathrm{a} 1+m \mathrm{a} 2$ is the helical vector ( $n$ and $m$ are integers), and the value is the girth of CNT, and the direction of the vertical helical vector $\mathrm{Ch}$ is the axial direction, the corner between $\mathrm{Ch}$ and al (the axial direction of sawtooth) is the helical angle $\theta$ (Paulmn S, 2000, P.1742 \& Kagotani T, 2004, P.1813).

Because of special structure, CNT has special performances, and good electrical and mechanical performances make CNT become the research hotspot with good foreground in the complex material domain (Zhang, 2000, P.1-4 \& FILIPPOV V, 1995, P.289 290 \& Cao, 1993, P.34-37 \& Zhao, 2002, P.220). The quantum size effect and the tunnel effect induced by the special structure of nanometer materials will induce many special performances, being different with traditional materials, and one the one hand, because the size of nanometer particle is in 
$1-100 \mathrm{~nm}$, which is far less than the wavelength of the electromagnetic wave emitted by the radar, the permeability of the nanometer materials to this wave is stronger than common materials, which could largely reduce the reflectivity of the wave, and the reflection signal received by the radar will become weak, and the stealth function will be achieved. On the other hand, the surface area of nanometer particle material is much thicker than common materials for 3 or 4 orders of magnitude, and it could absorb much more electromagnetic waves and infrared wave, and the electromagnetic wave emitted by the radar and the infrared wave emitted by the detected object will be adsorbed by the nanometer particles, which made the infrared detector and the radar to be difficult to detect the target. In addition, with the thinning of particles, the surface effect and the quantum size effect of the particles become very extrusive, and the interface polarization and the multiple dispersion of particles will be the important wave absorption mechanism, and the quantum size effect will make the electric power level of the nanometer particle to be cracked, and the interval is just in the range of the microwave energy, so new wave absorption channel will form (Zhao, 2002, P.220).

The SWCNT-paraffin wave absorption material is mainly introduced in this article. CNT is the one-dimensional nanometer material, and the small size effect, the quantum size effect, and the surface interface effect of nanometer particle make it have special characters of light, electron, magnetic, and noise, so the characters of CNT is different with common macro materials. But, because the magnetic dissipation of CNT is much less, which is disadvantageous for the wave absorption performance of microwave, to optimize the CNT complex materials as the wave absorption materials, it is very necessary to study the permeability of the nano-cluster or other nanometer structures. Because the wave absorption materials mean the materials which could absorb the electromagnetic wave energy casted on its surface, and change to heat energy by the dissipation. At present, the wave absorption materials made by the CNT have been widely researched and applied because of many advantages such as strong absorption, wide frequency bond, small proportion, think depth, and good environmental stability. According to the internal mechanism of the wave absorption materials, if the electromagnetic parameters such as the permeability $\left(\mu_{r}\right)$ and the dielectric constant $\left(\varepsilon_{r}\right)$ of the materials are confirmed, the wave absorption performance of the materials could be confirmed. At present, the key to enhance the wave absorption performance of the CNT covered wave absorption materials is to enhance the complex permeability of CNT and widen the wave absorption frequency direction, so it is very important to study the permeability direction of CNT. Therefore, the relationship between the wave absorption performance of CNT and the interior permeability is mainly discussed in this article, and the electromagnetic parameters of SWCNT materials with different frequencies under different concentrations are analyzed, and the corresponding exterior conditions of the optimal wave absorption performance are evaluated in the article.

\section{Experiment samples and testing}

The main sample in this experiment is the SWCNT which has not been disposed before it is used. The experiment data evolves in the samples of SWCNT and paraffin complex under five different proportions, and the complex dielectric constants and complex permeability in $2-18 \mathrm{GHz}$ are measured, and the permeability of SWCNT and paraffin complex is mainly studied in this article.

The coaxial-line method is used to measure the electromagnetic parameters of the SWCNT and paraffin complex. The electromagnetic wave emitter is the Hewlett Packaed 83860L series swept CW generator (HP83860L), and the emission frequency range is $10 \mathrm{MHz}-26.5 \mathrm{GHz}$, and the type of the network analyzer is the HP8722ES. When the coaxial-line method is used to measure the electromagnetic parameters of CNT, the pure CNT has large activity, and it is hard to form required shape, so it is very difficult to directly measure the electromagnetic parameters of the pure CNT, and when the CNT is used in the wave absorption materials, it will be composited with different matrix bases. The melting point of the paraffin is very low $\left(\sim 60^{\circ} \mathrm{C}\right)$, and the real part of the complex dielectric constant is basically unchangeable in $2-18 \mathrm{GHz}(2.23 \sim 2.27)$, and the imaginary part is in $0 \sim 0.01$, and the dielectric losses approach to 0 . So the paraffin is mainly used as the base to test the electromagnetic parameters of the absorption agent, and evaluate the wave absorption performance. CNTs are evenly decentralized in melting paraffin, and cast in the cooper loop standard flange, and the loop measured sample is formed which interior diameter is $3.0 \mathrm{~mm}$, which exterior diameter is $7.0 \mathrm{~mm}$, and which depth is $2.0 \mathrm{~mm}$. In the testing, the standard flange filled by the measured sample is linked with the testing system, and the testing system will directly provide the real part and the imaginary part of the complex dielectric constant and the complex permeability.

\section{Data processing and discussions}

\subsection{Software processing of data and data charting}

According to above experiment, the real parts and the imaginary parts of the electromagnetic parameters of the 
SWCNT-paraffin complex under five different concentrations in $2-18 \mathrm{GHz}$ are obtained respectively. The parameters of the permeability will be studied in this article. Because the experiment data are large, and the rule is hard to discover, so it needs the charting to help the analysis.

The software processing uses the function to simulate the scientific plotting software and the data analysis software, i.e. Origin software, and the obtained data are disposed respectively, and in $2-18 \mathrm{GHz}$, the permeability of SWCNT-paraffin complex is compared respectively, and the curve change under different concentrations is displayed, which could provide the theoretical reference to design new wave absorption materials.

\subsection{Frequency spectrum of the real part of the permeability of SWCNT complex}

The change curve of the real part $\mu^{\prime}$ of the permeability of the SWCNT in $2-18 \mathrm{GHz}$ is seen in Figure 3 , and $\mu^{\prime} 1-\mu^{\prime} 5$ respectively correspond with real part curve of the permeability of CNT under different concentrations of $5 \%, 8 \%, 10 \%, 15 \%$, and $20 \%$. In Figure 3, in $2-18 \mathrm{GHz}$, for these concentrations, the real parts of the permeability of SWCNT all approach to 1 , and when the concentration is $5 \%$, the corresponding curve is relatively smooth, and with the increase of the frequency, the value of $\mu^{\prime}$ changes a little and approaches to 1 . With the increase of concentration, for example, when the concentration is $15 \%$, the corresponding value of $\mu^{\prime}$ begins to fluctuate obviously, and the change tendency of $\mu^{\prime}$ will be gradually small, and when the concentration increases to $20 \%$, the change of $\mu^{\prime}$ will be very obvious with the increase of frequency, and the in the lower frequency range, the value of $\mu^{\prime}$ is less than 1 but approaches to 1 , and when the frequency increases, the value of $\mu^{\prime}$ begins to decrease quickly, and when the frequency is about $8 \mathrm{GHz}$, the minimum value of $\mu^{\prime}$ occurs and is about 0.83 , and here, when the frequency increases continually, the value of $\mu^{\prime}$ begins to increase drastically, and achieves about the maximum value, 1.17 , at the frequency of $18 \mathrm{GHz}$. So the change of the value of $\mu^{\prime}$ of SWCNT is related with the concentration, and when the concentration is lower (above 15\%), the value of $\mu^{\prime}$ is not related with the change of frequency. When the concentration increases, the value of $\mu^{\prime}$ begins to fluctuate with the increase of frequency, and when the concentration is higher, the tendency is more obvious, and both the maximum value and the minimum value of $\mu^{\prime}$ occur in this experiment under the maximum concentration of $20 \%$.

\subsection{Frequency spectrum of the imaginary part of the permeability of SWCNT complex}

Figure 4 is the change curve of the imaginary part $\mu^{\prime \prime}$ of the permeability in $2-18 \mathrm{GHz}$ with the change of frequency, and $\mu^{\prime \prime} 1-\mu^{\prime \prime} 5$ respectively correspond with the imaginary part curves of the permeability of CNT under the concentrations of $5 \%, 8 \%, 10 \%, 15 \%$, and $20 \%$. It can be observed that the value of $\mu^{\prime \prime}$ is basically in $-0.06-0.01$. For the lower concentrations (such as the $\mu^{\prime} 1$ curve and the $\mu^{\prime \prime} 2$ curve), the value of $\mu^{\prime \prime}$ increases with the increase of frequency, but when the frequency continually increases (exceeding $6 \mathrm{GHz}$ ), the value keeps stably, and when the concentration continually increases to $10 \%$, the value of $\mu^{\prime \prime}$ will increase continually with the increase of frequency. And when the concentration increases to $20 \%$, the value of $\mu^{\prime \prime}$ increases quickly (the maximum value is 0.03 ) in the lower frequency range (less than $6 \mathrm{GHz}$ ), and when the frequency exceeds the point of $6 \mathrm{GHz}$, the value of $\mu^{\prime \prime}$ decreases quickly, and when the frequency exceeds $10 \mathrm{GHz}$, the value of $\mu^{\prime \prime}$ keeps stably. In theory, $\mu^{\prime \prime}$ is the rearrangement loss which is induced by the magnetic dipole moment in the exterior magnetic field, and the imaginary parts $\varepsilon^{\prime \prime}$ and $\mu^{\prime \prime}$ of $\varepsilon_{r}$ and $\mu_{r}$ absorb the electromagnetic waves and induce the energy losses in the electrodynamics.

For good wave absorption materials, the value of $\tan \delta$ is bigger and better, i.e. the value of $\mu^{\prime \prime}$ is bigger and better, and the corresponding value of $\mu^{\prime}$ should be smaller. For the convenience, the ratio between the imaginary part and the real part of the permeability complex in same frequency is seen in Figure 5, and in $2-18 \mathrm{GHz}$, the change curve of the ratio between $\mu^{\prime \prime}$ and $\mu^{\prime}$ is seen in this Figure, and R1-R5 respectively correspond with the ratio curve between the real part and the imaginary part of CNT under the concentrations of $5 \%, 8 \%, 10 \%, 15 \%$, and $20 \%$.

\subsection{Frequency spectrum of the dissipation factor of the permeability of SWCNT complex}

The curve of Figure 5 is similar with the curve of Figure 4, that is because the value of $\mu^{\prime}$ under different concentrations seen in Figure 1 basically approaches to 1, and the curve $\mathrm{R}$ of the ratio taking the value of $\mu^{\prime}$ as the denominator is decided by the value of $\mu^{\prime \prime}$. The values of dissipation factor approximately centralize in -0.08-0.02. Being similar with Figure 2, for the lower concentrations (such as $\tan \delta 1$ curve and $\tan \delta 2$ curve), the value of $\tan \delta$ increases with the increase of frequency in lower frequency range, but it keeps stably when the frequency continually increases (exceeding $6 \mathrm{GHz}$ ), and when the concentration continually increases to $10 \%$, the value of $\tan \delta$ increases with the increase of frequency and continually increases. When the concentration 
increases to $20 \%$, the value of $\mathrm{R}$ increases quickly (the maximum value is 0.033 ) in the lower frequency range (less than $6 \mathrm{GHz}$ ), and when the frequency exceeds the point of $6 \mathrm{GHz}$, the value of $\tan \delta$ decreases quickly, and when the frequency exceeds $10 \mathrm{GHz}$, the value of $\mathrm{R}$ increases slowly. Considering the curves of $\mu^{\prime}$ and $\mu^{\prime \prime}$ and their ratio curve $\tan \delta$, the dielectric magnetic loss $\tan \delta_{M}=\left(\frac{\mu^{\prime \prime}}{\mu^{\prime}}\right)$ of CNT is influenced by the frequency and the concentration, so when choosing the wave absorption materials, the permeability should be considered comprehensively, and so the optimal permeability distribution bond should be fitted in the wider frequency range, and the ideal value of $\tan \delta$ could be obtained to enhance the wave absorption performance of the materials.

\section{Conclusions}

(1) In same concentration and frequency, the real parts of the permeability of SWCNT are almost same, and basically approach to 1 .

(2) The frequency spectrums of the real part, the imaginary part and the dissipation factor of the permeability of SWCNT all have similar changing tendency, i.e. when the concentration is lower, their values almost change nothing with the increase of frequency, and when the concentration is higher, their values change obviously with the increase of frequency, and the concentration is higher, their values will increase more obviously with the increase of frequency, and the maximum values all occurs in lower frequency and high concentration.

(3) In higher frequency range, the real part, the imaginary part, and the dissipation factor of the permeability of SWCNT change nothing with the change of frequency, and that is because the change of magnetic field influences the arrangement of the magnetic dipole, and the magnetic dipole will be lagged behind the change of the magnetic field in high frequency range.

Above conclusions indicate that in lower concentration, the real part of the permeability of SWCNT almost changes nothing with the change of the concentration and the frequency, and keeps tiny fluctuation, but in higher concentration, the real part of the permeability of SWCNT changes obviously with the change of frequency, and when the concentration is bigger, the fluctuation is more obvious and the maximum value will occur. But the imaginary part and the ratio between the real part and the imaginary part will present complex change rule with the change of frequency, and the total tendency could be described by that in lower concentration, the imaginary part and the ratio between the imaginary part and the real part change little with the change of frequency, and in the higher frequency, the value is sensitive to the change of frequency, and the maximum value occurs.

Because the main aspect to enhance the wave absorption performance of CNT is to enhance the complex permeability of CNT and widen the frequency length of wave absorption, it is very important to study the permeability direction of CNT (Cao, 2007, P.1-3 \& Xing, 2004, P.93), and the further works include that trying to enhance the contribution of the permeability and dissipation factor to the wave absorption by the further research of CNT complex, designing different exterior conditions to find the optimal fitting point of the permeability with the optimal wave absorption performance, improving and adjusting the dielectric constant and the complex permeability to strength the performance of wave absorption, studying the concrete interior pertinence of the magnetic dissipation factor and the concentration in special frequency, and providing theoretical reference for the practical design of new materials.

In conclusion, by studying the interior relationships between the different formations of wave absorption materials, the concrete influences of different conditions on the permeability and the wave absorption performance, the new wave absorption materials which could not only strengthen the absorption, but absorb the electromagnetic wave in the wide frequency range will certainly occur.

\section{References}

Bi, Hong, Wu, Xianliang \& Li, Minquan. (2005). Technics of Aerospace Materials. Beijing: National Defense Industrial Press. P.34.

Cao, Hui. (1993). Structural Radar Absorbing Materials and Applications. Technics of Aerospace Materials, No.4. P.34-37.

Cao, Wei, Song, Xuemei, Wang, Bo \& Yan, Hui. (2007). Research Development of Carbon Nano Tubes. Beijing: National Defense Industrial Press. P.1-3.

Ebbesen T W, Lezee H J, Hiura H, et al. (1996). Conductive properties of Carbon Nanotube/ epoxy composite material. Nature, No.382. P.54.

FILIPPOV V. (1995). Influence of a thin dielectric layer on nonreciprocal effects arising at reflection from 
magneto-optic films. Journal of Magnetic Materials, No.148(1-2). P.289 290.

Iijima S. (1991). Helical microtubules of graphitic carbon. Nature, No.354. P.56-86.

Kagotani T, Fujiwara D, Sugimoto S, et al. (2004). J Magn Magn.REE in the magnetic absorbing materials. Matter, No.272-276. P.1813.

Paulmn S, Helser A, Nardelli M. (2000). Carbon Nanotubes absorbing materials on the Status and Prospects. Science, No.290. P.1742.

Taurisano M D,Vorst A V.1EEE T. (2000). Microwave Theory, No.48(11). P.2022.

Xing, L. Y. (2004). Stealth materials. Beijing: Chemical Industry Press. P.93.

Zhang, Weidong, Feng, Xiaolan \& Meng, Xiulan. (2000). Status and Development of Foreign Study on New Stealthy Materials. Aerospace Materials \& Technology, No.3. P.1-4.

Zhao, Jiupeng, Li, Yao \& Wu, Peilian. (2002). Recent Developments of Studies on New Electromagnetic Wave Absorbing Materials. Materials Science and Technology, No.2. P.220.

Zhong, Hua \& Li, Zili. (1999). Anti-stealth Technology. Beijing: National Defense Industrial Press. P.26-30.

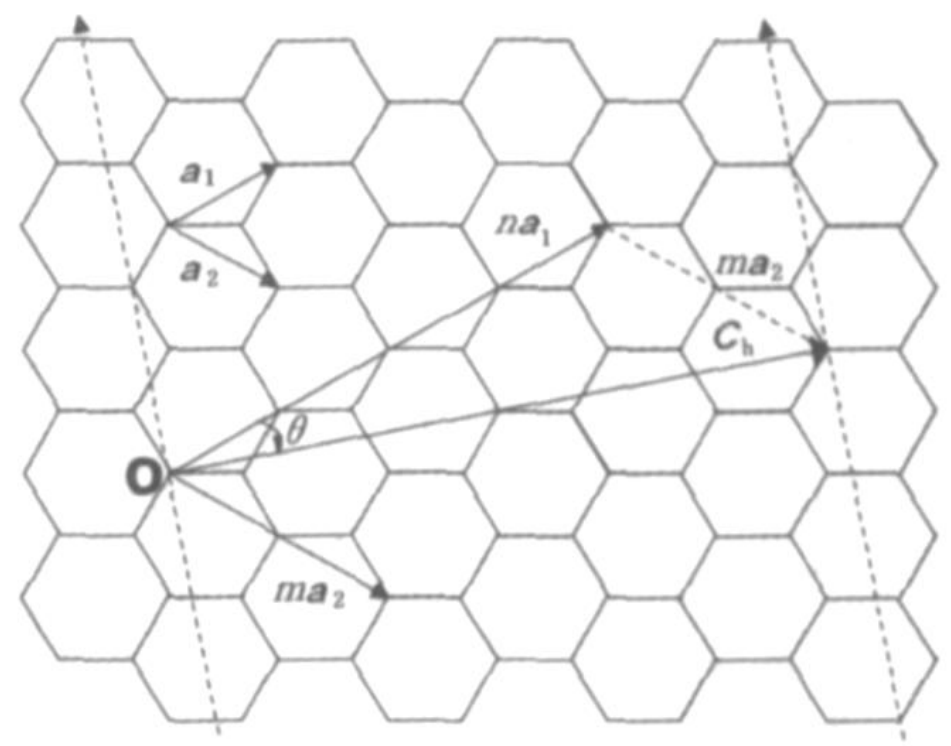

Figure 1. Geometric Meanings of CNT Parameters (Kagotani T, 2004, P.1813)

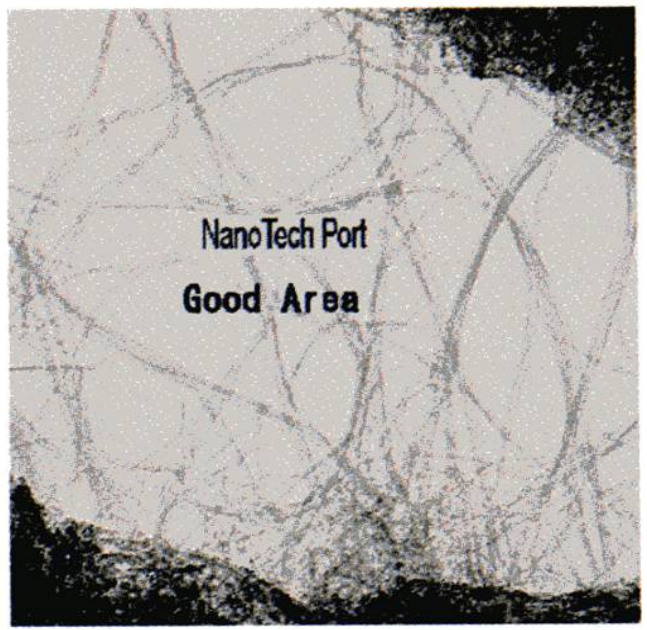

Figure 2. SWCNT 


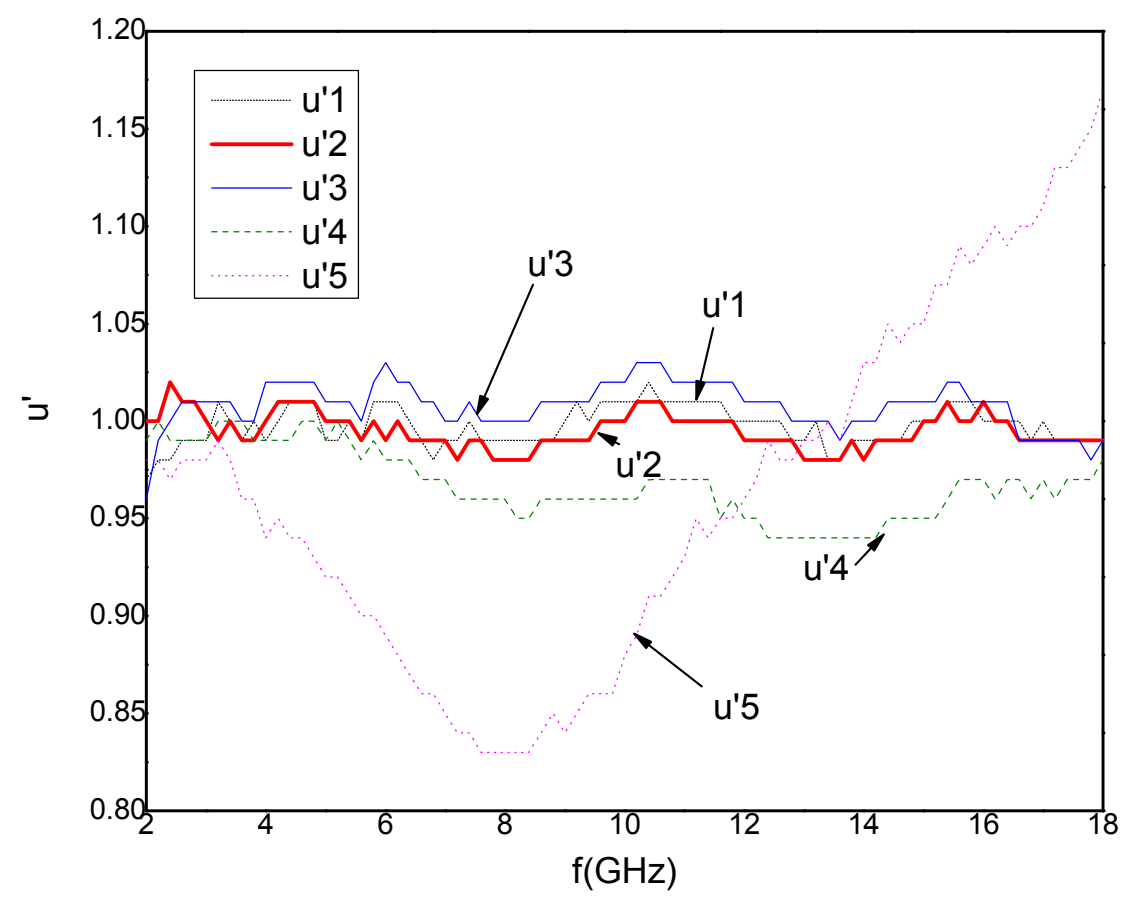

Figure 3. The Frequency Spectrum of the Real Part of the Permeability of SWCNTs under Five Concentrations in $2-18 \mathrm{GHz}$

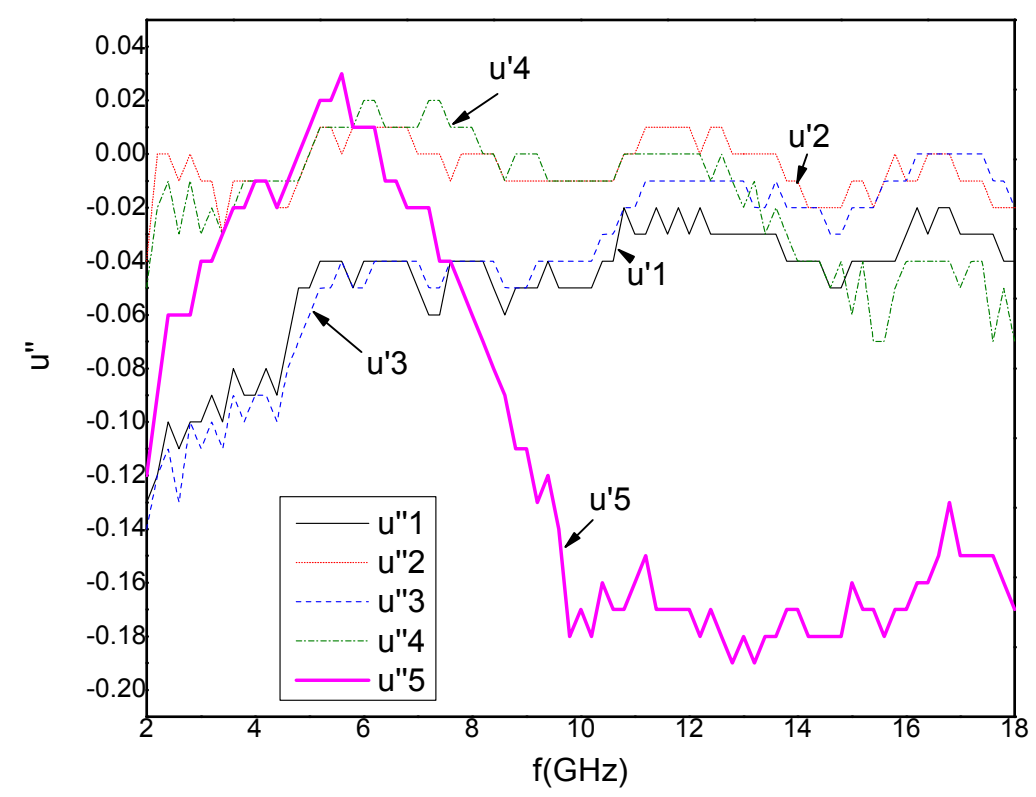

Figure 4. The Frequency Spectrum of the Imaginary Part of the Permeability of SWCNTs under Five Concentrations in $2-18 \mathrm{GHz}$ 


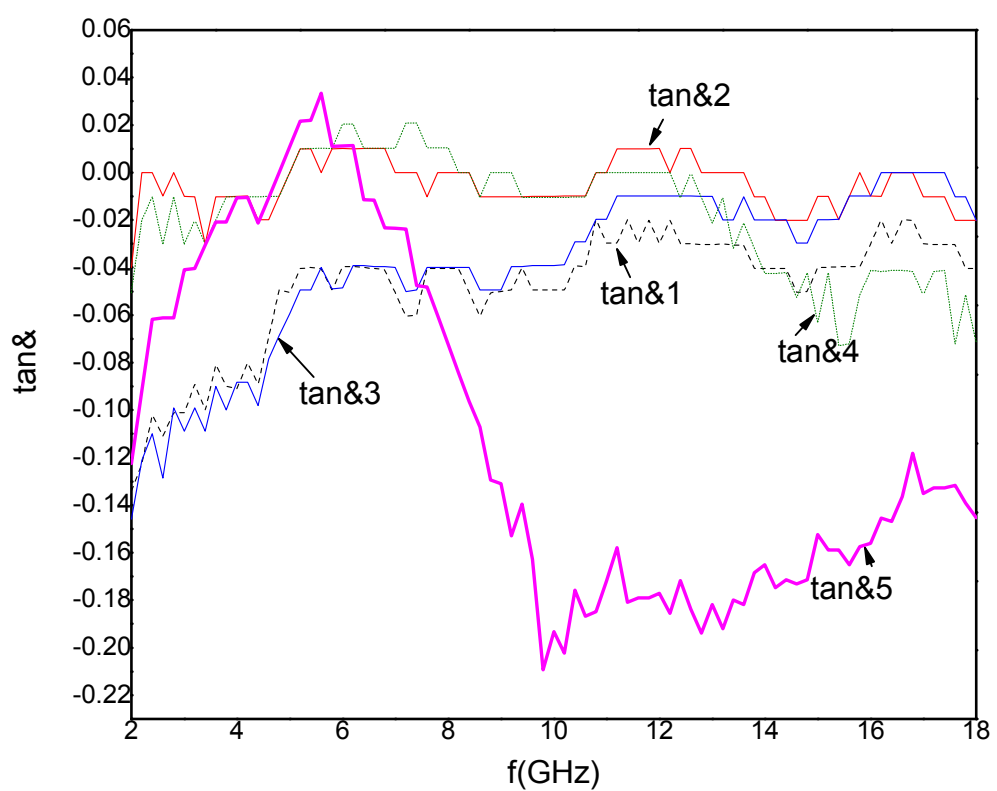

Figure 5. The Frequency Spectrum of the Dissipation Factor of the Permeability of SWCNTs under Five Concentrations in $2-18 \mathrm{GHz}$ 